【研究简报】

\title{
含 $\mathrm{La}$ 的 SiMCM-41 的合成及其水热稳定性
}

何农跃鲍书林须沁华

(南京大学化学系, 南京 210093)

\section{关链词 LaSiMCM-41 合成 表征 水热稳定性}

1992 年 Mobil 公司首次报道了孔径在 2 10 nm 之间的人工合成中孔 MCM-41 系分子 篣 ${ }^{[1]}$, 无论作为催化剂还是主客体化学的主体, 都有着诱人的应用前景. 但目前需要解决的 重要问题之一是提高其水热稳定性. 本文首次研究了将稀土 La 引入中孔 SiMCM-41 中的合 成, 并讨论其水热稳定性, 实验结果表明 $\mathrm{La}$ ( III ) 的引入可明显地改善 MCM-41 的水热稳定性.

\section{1 实验}

\section{1 样品制备}

以水玻璃 $\left(20.3 \% \mathrm{SiO}_{2}, 6.7 \% \mathrm{Na}_{2} \mathrm{O}\right), \mathrm{C}_{16} \mathrm{H}_{33}\left(\mathrm{CH}_{3}\right)_{3} \mathrm{NBr}(\mathrm{CTAB}), \mathrm{La}\left(\mathrm{NO}_{3}\right)_{3}$ 为原料, 配制 摩尔组成为 $1.0 \mathrm{SiO}_{2}: x \mathrm{La}_{2} \mathrm{O}_{3}: 0.18 \mathrm{Na}_{2} \mathrm{O}: 0.25 \mathrm{CTAB}: 57 \mathrm{H}_{2} \mathrm{O}$ 的反应混合物, 其中 $x$ 在 $0 \sim$ 0.03 之间变动. 当 $x=0$ 时, 得样品 SiMCM-41, 其余含 $\mathrm{La}$ 样品用 ( $\mathrm{La}$ ) SiMCM-41 表示. 将 所得反应混合物转入内衬为聚四氟乙烯的不锈钢高压釜中, 在 $373 \mathrm{~K}$ 下反应 $7 \mathrm{~d}$, 冷至室温, 抽 滤洗涤并经室温干燥后即得样品. (La) AlSiMCM-41 及 AlSiMCM-41 采用类似的方法合成. 将样品在 $813 \mathrm{~K}$ 下于氮气氛中处理 $1 \mathrm{~h}$, 再在空气氛中处理 $4 \mathrm{~h}$ 除去模板剂后进行表征.

\section{2 样品表征}

样品表征在 HRTEM(高分辩透射电子显微镜) 在 JEOLJEM- 200 CX 上测定. 骨架 IR(红 外)谱在 Nicolet 5DX FT-IR 仪上测定. 小角 XRD (X 射线衍射)在 Rigaku D/Max-rA 上测定. 低温氮吸附等温线在 Micromeritics ASAP 2000 上测定. 以吸附苯的相对比值考察样品的水 热稳定性.

\section{2 结果与讨论}

\section{1 样品的结构}

在实验所示凝胶组成范围内都可得到中孔 MCM-41 分子篣, 表 1 列出了几个典型样品的 反应凝胶组成及除去模板剂后样品的表征结果.

表 1 样品的凝胶组成及焙烧后样品的物化性质 ${ }^{\mathrm{a})}$

\begin{tabular}{|c|c|c|c|c|c|c|c|c|c|}
\hline \multirow[b]{2}{*}{ 样品 } & \multicolumn{2}{|c|}{ 凝胶组成 $/ \mathrm{mol}$} & \multicolumn{7}{|c|}{ 焙烧后样品的组成及其物化性质 } \\
\hline & $\frac{\mathrm{SiO}_{2}}{\mathrm{La}_{2} \mathrm{O}_{3}}$ & $\frac{\mathrm{SiO}_{2}}{\mathrm{Al}_{2} \mathrm{O}_{3}}$ & $\frac{\mathrm{SiO}_{2}}{\mathrm{La}_{2} \mathrm{O}_{3}}$ & $\frac{\mathrm{SiO}_{2}}{\mathrm{Al}_{2} \mathrm{O}_{3}}$ & $\begin{array}{l}d_{100} \\
/ \mathrm{nm}\end{array}$ & $\begin{array}{l}a_{0} \\
/ \mathrm{nm}\end{array}$ & $\begin{array}{l}\text { 比表面 } \\
/ \mathrm{m}^{2} \cdot \mathrm{g}^{-1}\end{array}$ & $\begin{array}{c}\text { 孔直径 } \\
(\mathrm{BJH}, \mathrm{nm})\end{array}$ & $\begin{array}{c}\text { 孔壁厚 } \\
/ \mathrm{nm}\end{array}$ \\
\hline SiMCM-41 & pure & siliceous & & & 4.032 & 4.656 & 1340 & 3.169 & 1.487 \\
\hline (La) SiMCM-41 & 66 & - & 83 & - & 4.084 & 4.716 & 932 & 3.262 & 1.454 \\
\hline (La) AlSiMCM-41 & 66 & 66 & 116 & 40 & 4.193 & 4.842 & 944 & 3.062 & 1.780 \\
\hline AlSiMCM-41 & - & 66 & - & 58 & 4.151 & 4.793 & 1084 & 3.257 & 1.536 \\
\hline
\end{tabular}

a) $a_{0}=2 d_{100} / \sqrt{3}$, 孔壁厚 $=a_{0}-$ 孔直径 
小角 XRD 清楚地表明 (图 1), 各样品除了在 $2 \theta=2 \sim 3^{\circ}$ 之间有一个很强的 (100) 面衍射峰 外, 在 3 $6^{\circ}$ 之间还可观察到 2 或 3 个小峰, 与文献 [1]所报道是一致的, 因而所得样品为六角 形相的 MCM-41 分子篮, 晶胞参数 $a_{0}$ 一并列于表 1 中, $a_{0}$ 值也就是相邻两孔道间的距离 ${ }^{[2]}$. 由表 1 可看到, 所制备的 (La) SiMCM-41 的晶胞参数比 SiMCM-41 略大. 对 (La) SiMCM-41 样品进行 HRTEM 观察(图 2), 可清楚地看到样品呈六边形排列的孔道 ${ }^{[1]}$. (La) SiMCM-41

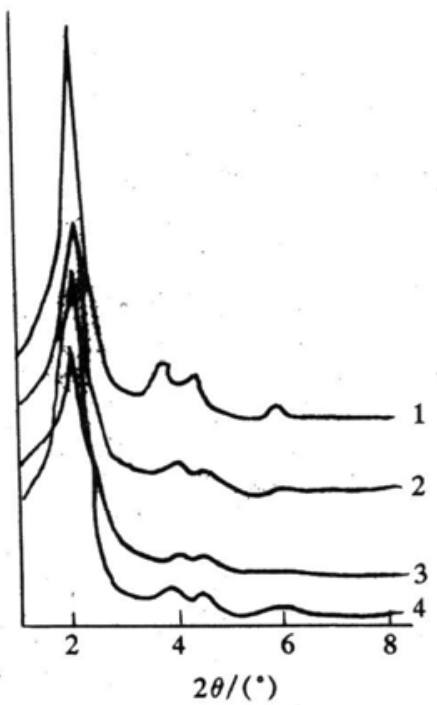

图 1 样品的 XRD 图 1 为 $\mathrm{SiMCM}-41,2$ 为 $(\mathrm{La}) \mathrm{SiMCM}-41,3$ 为 (La) AlSiMCM-41, 4 为 AlSiMCM-41

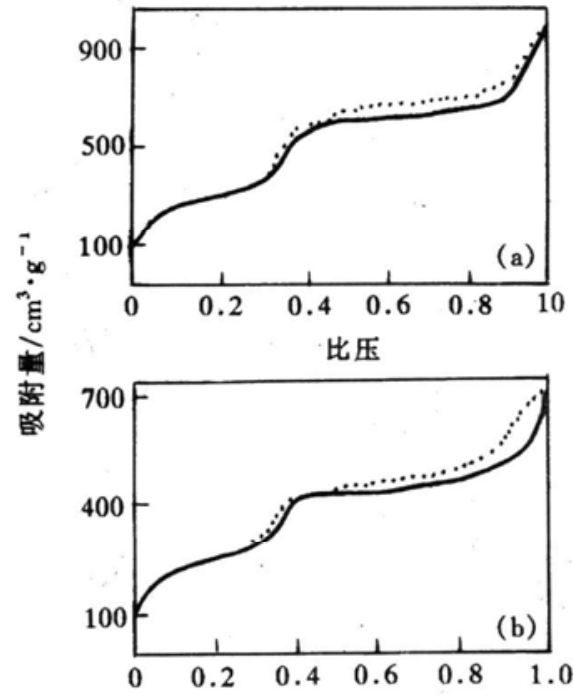

图 3 样品的 $\mathrm{N}_{2}$ 吸附等温线 (a) SiMCM-41, (b) (La)SiMCM-41
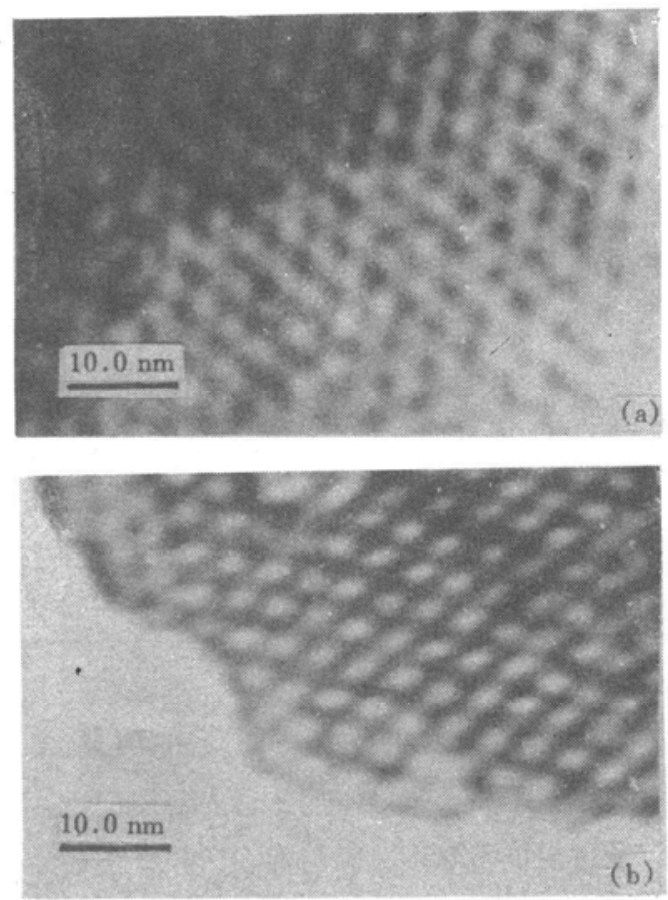

图 2 样品的 HRTEM 照片

(a) SiMCM-41, (b) (La)SiMCM-41

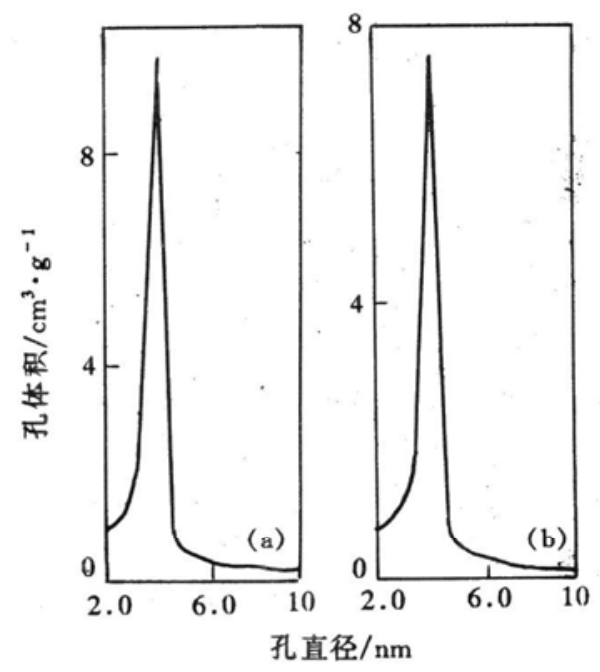

图 4 样品的孔径分布

(a) SiMCM-41, (b) (La)SiMCM-41 
样品的骨架 FT-IR 谱与无定形 $\mathrm{SiO}_{2}$ 类似 (图略), 说明样品的孔壁为无定形. (La) SiMCM-41 样品的 $N_{2}$-BET 吸附等温线 (图 3) 在 $P / P_{0}$ 为 $0.35 \sim 0.40$ 之间出现一个突跃, 这是 $N_{2}$ 分子在 中孔孔道内发生毛细凝聚的结果 ${ }^{[3]}$. 在该突跃处伴随有一个小的滞后环, 表明中孔直径在 3 $\mathrm{nm}$ 以上 ${ }^{[3]}$. 图 4 为由图 3 的吸附等温线按 $\mathrm{BJH}$ 模型计算出的样品孔径分布图. 由图可见样 品的孔分布集中在约 $3.3 \mathrm{~nm}$ 处, 表明孔道的均匀性. 整个中高比压部分较大的滞后环则可能 是较大孔径的次级中孔或粒子间的中孔所致 ${ }^{[3]}$.

\section{2 样品的水热稳定性}

由表 2 可见, 尽管水热处理前各样品的苯吸附量都较大, 但水热处理后 (La) SiMCM-41 的苯吸附量保留值 $\left(c / c_{0}\right)$ 比 SiMCM-41 和 AlSiMCM-41 都要大, 由此相对比值得出的水热稳 定性顺序为: (La) SiMCM-41>SiMCM-41> ( La) AlSiMCM-41>AlSiMCM-41.

表 2 样品在水热处理前后的苯吸附量 ${ }^{\text {( ) }}$

\begin{tabular}{lcccc}
\hline \multirow{2}{*}{ 样品 } & 孔壁厚度 & \multicolumn{2}{c}{ 苯吸附量 } & \multirow{2}{*}{$c / c_{0} / \%^{b}$ ) } \\
\cline { 3 - 4 } & $/ \mathrm{nm}$ & 水热处理前 $/ \mathrm{g}^{\circ} \cdot \mathrm{g}^{-1}$ & 水热处理后 $/ \mathrm{g} \cdot \mathrm{g}^{-1}$ & \\
\hline SiMCM-41 & 1.487 & 0.680 & 0.381 & 56 \\
(La)SiMCM-41 & 1.454 & 0.548 & 0.348 & 64 \\
(La)AlSiMCM-41 & 1.780 & 0.465 & 0.234 & 50 \\
AlSiMCM-41 & 1.536 & 0.655 & 0.278 & 42 \\
\hline
\end{tabular}

a) 水热处理条件: $933 \mathrm{~K}$, 饱和水蒸汽, $2 \mathrm{~h} ; \mathrm{b}) c_{0}, c$ : 水热处理前后苯吸附量 $(298 \mathrm{~K}$ )

通常 MCM-41 的水热稳定性与孔壁厚度有关, 孔径相同时, 孔壁越厚, 稳定性越好 ${ }^{[2]}$. 但 实验表明 (见表 2), 虽然 (La)AlSiMCM-41 及 AlSiMCM-41 具有比 SiMCM-41 和 (La)SiMCM41 较大的孔壁厚度, 水热稳定性却很差, 表明 $\mathrm{Al}$ 的引入降低了水热稳定性. ( $\mathrm{La}$ ) SiMCM-41 虽然孔壁厚度最小, 水热稳定性却最好, 表明 $\mathrm{La}$ ( III) 的引入可提高样品的水热稳定性, 即在水 热处理过程中 $\mathrm{La}$ (III) 也具有稳定 MCM-41 孔壁的作用,但与 $\mathrm{Al}$ 共同引入时这种稳定作用被 削弱.

综上所述,在我们实验条件下,我们首次制得了含 $\mathrm{La}$ 的孔径约为 $3.3 \mathrm{~nm}$ 的六角形相的 (La)SiMCM-41 中孔分子篮, 其水热稳定性明显地优于 SiMCM-41 和 AlSiMCM-41.

致谢 本工作为国家自然科学基金(批准号:29133078) 资助项目.

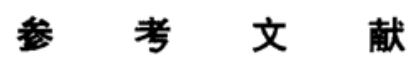

1 Kresge C T, Leonowicz M E, Roth W J, et al. Ordered mesoporous molecular sieves synthesized by a liquid-crystal template mechanism. Nature, 1992, 359:710 712

2 Feuston B P, Higgins J B. Model structures for MCM-41 materials: a molecular dynamics simulation. J Phys Chem, 1994, $98: 4459 \sim 4462$

3 Rathousky J, Zukal A, Franke O, et al. Adsorption on MCM-41 mesoporous molecular sieves. J Chem Soc, Faraday Trans, 1994, $90: 2821 \sim 2826$ 